\title{
Efecto de la procedencia y el almacenamiento en en la calidad del café
}

\author{
Effect of provenance and storage on coffee quality
}

Muñoz-Juan Carlos ${ }^{1}$, Riaño-Campo Elías², Pinzón-Alba Janeth ${ }^{3}$

\section{Resumen}

Antecedentes. La calidad del grano de café ha sido relacionada con su procedencia, su manejo agronómico y sus condiciones de almacenamiento. Objetivo. Determinar la actividad de la polifenil oxidasa, el contenido de lípidos, el color y las características organolépticas de cafés provenientes de 3 subestaciones experimentales. Materiales y métodos. Se siguió un diseño completamente aleatorio en arreglo factorial factorial 3x6 (lugares de procedencia del café y tiempo de almacenamiento respectivamente). Resultados. La actividad de la polifenil oxidasa es mayor en el café fresco-para las tres procedencias. El café procedente de Naranjal presentó actividades enzimáticas más altas que los cafés provenientes de las subestaciones Supía y la Catalina. El análisis de varianza mostró el efecto de la procedencia sobre la variable actividad enzimática. La actividad de la polifenil oxidasa en los cafés estudiados decrece con el tiempo de almacenamiento. El contenido de lípidos es menor a menor en la subestación de la Catalina. Todos los cafés fueron caracterizados de buena calidad en el tiempo cero de almacenamiento. Las características de aroma, intensidad del aroma y cuerpo presentaron altibajos en los diferentes meses de almacenamiento. El café de Naranjal, obtuvo en promedio una calificación aceptable a lo largo de los seis meses de almacenamiento. Conclusiones. Se encontraron diferencias significativas para las variables estudiadas por efecto de la procedencia y el almacenamiento. La actividad enzimática de la PFO presentó etapas de activación/inhibición, durante los seis meses de almacenamiento.

Palabras claves: localización geográfica, calidad del café, almacenamiento, polifenil oxidasa, actividad enzimática, color.

1. Ingeniero Químico, Centro Nacional de Investigaciones sobre Café-CENICAFE. jcmtgc@gmail.com

2. Doctor en Educación en Tecnología Instruccional y Educación a Distancia de Nova Southeastern University - NSU, Florida - USA. Magister en

Ciencias de la Ingeniería, Universidad de los Andes - Colombia. Ingeniero Químico, Universidad de América. Docente asociado de la ECBTI de la UNAD - Universidad Nacional Abierta y a Distancia. Integrante del Grupo de Investigación Social-GIS, de la UNAD. campo.riano@unad.edu.co ORCID: https://orcid.org/0000-0002-3226-5332

3. MSc, Ingeniero Químico, Universidad Nacional de Colombia. Docente ocasional de la ECBTI de la UNAD- Universidad Nacional Abierta y a

Distancia. Integrante del Grupo de Investigación Social-GIS, de la UNAD. alba.pinzon@unad.edu.co

ORCID: https://orcid.org/0000-0002-6378-4434

Bogotá, Colombia. 


\section{Abstract}

Background. The quality of the coffee bean has been related to its origin, the agronomic management and the storage conditions. Objective. To determine the activity of the polyphenyl oxidase, the lipid content, the color and the organoleptic characteristics of coffees from 3 experimental substations. Materials and methods. A completely randomized design was followed in a $3 \times 6$ factorial arrangement (places of coffee origin and storage time respectively). Results. The activity of polyphenyl oxidase is greater in fresh coffee-for the three provenances. The coffee from Naranjal presented higher enzymatic activities than the coffees from the Supía and the Catalina substations. The analysis of variance showed the effect of provenance on the enzyme activity variable. The activity of the polyphenyl oxidase in the coffees studied decreases with storage time. The lipid content is lower at a lower height in the Catalina. All coffees were characterized as good quality at zero storage time; but the characteristics of aroma, intensity of aroma and body presented ups and downs in the different months of storage. Naranjal coffee, on average, obtained an acceptable rating throughout the six months of storage. Conclusions. Significant differences were found for the variables studied due to the effect of provenance and storage. The enzymatic activity of the PFO showed activation / inhibition stages, during the six months of storage.

Keywords: geographic location, coffee quality, storage, polyphenol oxidase, enzymatic activity, color.

\section{Introducción}

Para la Organización de las Naciones Unidas para la Alimentación y la Agricultura (FAO); un producto es de mejor calidad cuando es superior a otro en uno o varios atributos que son valorados objetiva o subjetivamente $(1,2$, $3)$. Varios autores $(4,5)$ han estudiado esta implicación en el grano de café. Por ejemplo, en $(6,7,8,9,10$, ) se estudiaron la influencia de las condiciones ambientales, edafològicas y la irrigación en las características químicas y bioquímicas. También se ha encontrado que la actividad de la enzima polifenil oxidasa (ActPPO) depende de las condiciones de procesamiento [despulpado, tiempo de fermentación, especie de café, temperatura de secado y almacenamiento] $(11,12,13)$. Otra sustancia asociada a la calidad del café son los lípidos, los cuales en promedio son el $15 \%$ del peso del grano $(13,14) y$, son los que transportan los sabores y aromas del café $(14,15)$. Igualmente, en (16) se diserta sobre la importancia y la responsabilidad de los compuestos fenólicos, en las características organolépticas, particularmente en el color y el gusto. Estos compuestos durante la tostación incrementan el amargo, la astringencia y potencian el aroma característico del café (17). De manera que se puede intuir que los compuestos fenólicos, como la polifenil oxidasa tienen relación con la calidad de la bebida de café. Otra propiedad física es el color del grano el cual es un referente de la frescura, el contenido de humedad, el nivel de la homogeneidad de las muestras y la autenticidad del producto (10, 
18, 19). Además en estudios sobre el color del grano se encuentra que este cambia con el entorno del cultivo o procedencia $(20,10)$.

Sin embargo, en las anteriores investigaciones no se reporta el efecto de las condiciones del entorno de cultivo, como la: temperatura, humedad, brillo solar y el almacenamiento de los granos sobre la ActPPO, el contenido de lípidos, el color, la acidez y las características organolépticas del café colombiano.

\section{Materiales y métodos}

Procedencia. Las subestaciones experimentales fueron: a) Naranjal [Latitud $4^{\circ} 59^{\prime}$ Norte, Longitud $75^{\circ} 39^{\prime} \mathrm{W}$, Altitud 1400 metros sobre el nivel del mar, temperatura promedio (Tpr) de $21.6^{\circ} \mathrm{C}$; ; b) Supía [Latitud $5^{\circ} 28^{\prime}$ Norte, Longitud 75³9' W, Altitud 1320 metros sobre el nivel del mar, Tpr de $22.3^{\circ} \mathrm{C}$ ], y c) la Catalina [ Latitud $4^{\circ} 45^{\prime}$ Norte, Longitud $75^{\circ} 44^{\prime}$ W, Altitud 1310 metros sobre el nivel del mar, Tpr de $21.6^{\circ} \mathrm{C}$ ]. Las 3 subestaciones emplean practicas artesanales y su producción es limpia, la recolección de los granos maduros se hace de manera manual, el beneficio es húmedo con agua de fuentes naturales y secado a la luz del sol de forma artesanal.

Las muestras. Cafés variedad Colombia de una misma cosecha y de las subestaciones en estudio.

Diseño experimental. Los tratamientos consistieron en tres 3 subestaciones experimentales de CENICAFE y 6 tiempos de almacenamiento (entre 1 y 6 meses) evaluados bajo un diseño experimental completamente aleatorio en arreglo factorial $3 \times 6(21)$.
Unidad experimental. 500 gramos de café crudo de cada una de las subestaciones anteriormente descritas. De cada uno de ellos se pulverizaron, y se dividieron en partes iguales para aplicarles los protocolos analíticos; las mediciones se realizaron antes de los 5 minutos-con el fin de evitar la oxidación del café $(5,15,16)$.

Variables de respuesta. La ActPPO fue cuantificada espectrofotométricamente; utilizando como substrato, DOPA (3,4 - dihidroxifenilalanina) la cual ha sido utilizada en la mayoría de los trabajos sobre PPO y calidad de la bebida $(4,16)$. La primera lectura de la actividad fue considerada como la lectura inicial o tiempo cero, valor que se le descuenta a los valores obtenidos de las muestras en estudio y analizadas a diferentes tiempos de almacenamiento para este trabajo.

El color se midió en un colorímetro Tristimulus HUNTER LAB DP9000, en escala de luminosidad (L) [0 negro _ 100 blanco], cromaticidad a [opacidad- a + rojo, a -verde], cromaticidad b [brillo_b + amarillo, b - azul] (22). Ver figura 1. 
Figura 1. El espacio de color CIELAB.

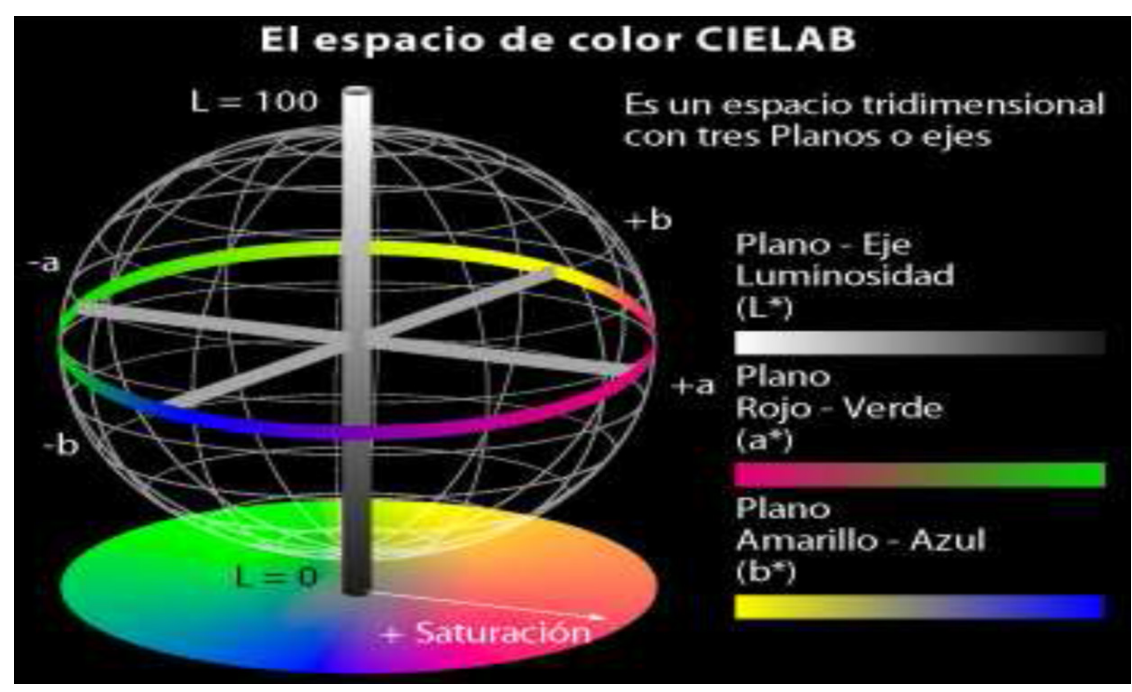

Fuente. Elaboración propia.

Se obtuvieron las coordenadas de color del CIEL*a*b, las cuales son un indicador de $\mathrm{L}^{*} \mathrm{o}$ la luminosidad, de $\mathrm{a}^{*}$ o de la cromaticidad [verde (-) a rojo (+)] y b* o cromaticidad azul $[(-)$ a amarillo $(+)]$. Para la medición se tomaron 10 gramos de café de cada tratamiento. Para calcular las diferencias de color, se utilizaron las relaciones matemáticas de la CIE y la CIELAB, donde la diferencia de color se representa por $\Delta E^{*}$ ab y la métrica de CIEDE, donde la diferencia de color se representa por $\Delta \mathrm{E}^{*} 00(23,24,25,26)$.

La diferencia de color normalizada $(\Delta \mathrm{E})$ se calcula acorde con la ecuación $1(25,26)$. $\Delta \mathrm{E}^{*}=\left[\Delta \mathrm{L} 2+\Delta \mathrm{a}^{*} 2+\Delta \mathrm{b}^{*} 2\right]$

\section{Ecuación 1}

En esta ecuación $\Delta \mathrm{E}^{*}$ es siempre positiva y es estrictamente la medida de la diferencia total de color entre el estándar (en este caso el café fresco de cada sitio) y la muestra proveniente del tratamiento o meses de almacenamiento. Solo si la diferencia del valor, sea positiva o negativa, nos dirá si la muestra del ensayo es más clara o más oscura que la referencia. Este realmente expresa la dirección de la diferencia de color.

Los otros parámetros físicos y químicos fueron determinados siguiendo los métodos (27) y de (28) para determinar la densidad por el método de densidad a granel por caída libre de los granos enteros, la humedad, $\mathrm{pH}$ y la acidez titulable de la bebida, lípidos y proteína.

Variables no paramétricas. Las cualidades organolépticas de los cafés provenientes de las 3 localidades fueron analizadas por el panel de CENICAFE en cuanto a la aroma, el color, el sabor, la textura y la aceptabilidad (29). Todas las evaluaciones organolepticas, fueron hechas por triplicado. Se les realiza un análisis descriptivo cuantitativo, en donde cada muestra fue evaluada individualmente, utilizando la lista de descriptores previamente definidas en (29). La intensidad de la percepción se indicó en la escala de percepción 
del descriptor, dentro del universo de muestras consideradas como lo trabajan en (29). Para las pruebas descriptivas cuantitativas se usó la escala de 9 puntos, desarrollada en CENICAFÉ para la calificación de cada característica organoléptica del café, la cual se interpreta así: calificaciones 9, 8, 7 para cualidades equilibradas, deseables, aceptables; 9 lo mejor; 6, 5, 4 califica desviaciones, 4 apenas tolerable; 3, 2, 1 califican defectos, rechazo y, por último, 1 , lo peor.

Análisis estadístico. Los datos obtenidos de los análisis químicos, físicos y bioquímicos de las variables estudiadas fueron analizados por análisis de varianza (ANAVA) y cuando la razón de Fisher (F) fue significativa, las medias fueron comparadas usando la prueba de diferencia mínima significativa (LSD) con una significancia del $5 \%(21)$.
Los datos fueron digitalizados en una planilla STATGRAPHICS 5 y los resultados se expresan en ANAVAs y LSD $(\mathrm{p}<0.05)$.

\section{Resultados y discusión}

\section{Diferenciación de los cafés en estudio.}

La estadística mostró que los resultados estaban dentro de los límites de confianza y rangos estipulados para cada variable, excepto para el color y la acidez titulable como se sustenta en otros trabajos $(6,8,30)$. Además, se obtuvieron altos coeficientes de variación para las variables actividad enzimática y cromaticidad $a^{*}$ estando en la dirección de los trabajos de varios autores (31). (Ver Tabla 1).

Tabla 1. Estadística descriptiva para las variables estudiadas en cafés crudos de tres procedencias y para tres tiempos de almacenamiento.

\begin{tabular}{|c|c|c|c|c|c|}
\hline \multirow{2}{*}{ Variable } & \multirow{2}{*}{ Media* } & \multirow{2}{*}{$\begin{array}{l}\text { Coeficiente de } \\
\text { variación }\end{array}$} & \multicolumn{2}{|c|}{ Intervalo de confianza } & \multirow{2}{*}{$\begin{array}{l}\text { Valores } \\
\text { reportados } \\
\text { en la } \\
\text { bibliografía }\end{array}$} \\
\hline & & & Linf & Lsup & \\
\hline $\begin{array}{l}\text { Activ_enzimática } \\
\text {-PPO (UA/min-g) }\end{array}$ & 66.170 & 80.58 & 56.770 & 75.571 & $55.99-67.66$ \\
\hline Humedad (\%) & 10.526 & 9.65 & 10.347 & 10.705 & $10-13 \%$ \\
\hline $\mathrm{pH}$ & 5.081 & 1.27 & 5.069 & 5.092 & 5.0 \\
\hline $\operatorname{Acidez}(\mathrm{ml} \mathrm{NaOH} 0.1 \mathrm{~N})$ & 10.23 & 18.44 & 9.90 & 10.56 & $15-20$ \\
\hline Proteína (\%) & 14.284 & 5.67 & 14.141 & 14.427 & $11-16$ \\
\hline Luminosidad ${ }^{* *}$ & 38.371 & 2.20 & 38.222 & 38.520 & 64.98 \\
\hline Cromaticidad (a)** & 0.007 & 7196 & -0.087 & 0.102 & 0.45 \\
\hline Cromaticidad $(\mathrm{b})^{* *}$ & 11.118 & 5.04 & 11.019 & 11.217 & 13.5 \\
\hline Lípidos (\%) & 14.025 & 6.08 & 13.874 & 14.175 & $14-18$ \\
\hline Densidad (g/cc) & 0.658 & 3.17 & 0.654 & 0.661 & $0.69-0.73$ \\
\hline \multicolumn{6}{|c|}{$\begin{array}{l}\text { * Son los valores promedio entre tratamientos para los seis meses de almacenamiento. } \\
\text { Todos los datos son el promedio }(\mathrm{n}=6) \text {. } \\
\text { ** Valores para el grano entero. }\end{array}$} \\
\hline
\end{tabular}

Fuente. Elaboración propia basada en los datos recolectados. 
El pardeamiento enzimático permite estudiar la evolución del cambio de color de un tejido vegetal por acción de la polifenoloxidasa en el tiempo, como lo propusieron $(30,33)$. De aquí, se infiere que las determinaciones de los cambios de coloración del café durante el almacenamiento resultan en cambios de los valores $\mathrm{L}^{*}, \mathrm{a}^{*} \mathrm{y} \mathrm{b}^{*}$, con los cuales se calculó el cambio $\Delta \mathrm{E}$ para cada procedencia en valores CIE L*a*b* como lo proponen $(23,24)$. El análisis de varianza (21) mostró el efecto de la procedencia sobre la variable cromaticidad (a) [donde a negativo (-) tendencia al verde y a positivo (+) tendencia al rojo), como se muestra en la tabla 2.

Tabla 2. Análisis de varianza para la cromaticidad de café crudo bajo tres fuentes de variación_procedencias y tiempos de almacenamiento.

\begin{tabular}{|c|c|c|c|c|c|}
\hline Fuente & $\begin{array}{l}\text { Suma de } \\
\text { Cuadrados }\end{array}$ & G libertad & $\begin{array}{c}\text { Cuadrados } \\
\text { medios del } \\
\text { error }\end{array}$ & $\begin{array}{l}\text { Razón F } \\
\text { calculada }\end{array}$ & $\begin{array}{l}\text { Razón F } \\
\text { tabulada }\end{array}$ \\
\hline \multicolumn{6}{|l|}{ Efectos Principales } \\
\hline A: Color: Altura-procedencia & 1,68997 & 2 & 0,844986 & 11,27 & 3.89 \\
\hline $\begin{array}{l}\text { B:Color:tiempo de } \\
\text { almacenamiento }\end{array}$ & 0,3816 & 6 & 0,0636 & 0,85 & 3,0 \\
\hline Residuos & 0,899629 & 12 & 0,074969 & & \\
\hline Total (corregido) & 2,9712 & 20 & & & \\
\hline
\end{tabular}

Fuente. Elaboración propia basada en los datos recolectados.

Puesto que el valor-p de la prueba-Ftabulada es menor que el obtenido para 0,05 existe una diferencia estadísticamente significativa entre las cromaticidades de color, las procedencias y la altura, con un nivel del $95.0 \%$ de confianza. No hubo diferencias significativas entre los tiempos de almacenamiento (Ftabulado mayor). Se puede decir que existe evidencia estadística suficiente para rechazar la hipótesis nula (Ho1); por lo tanto, se favorece la hipótesis alterna(Hi1), que indica que por lo menos un tratamiento es diferente. Para determinar cuáles medias son significativamente diferentes de otras, se utilizó el método de prueba de rangos múltiples (LSD) (21). Con este método hay un riesgo del 5,0\% al decir que cada par de medias es significativamente diferente, cuando la diferencia real es igual a 0 . La prueba de LSD muestra que el café de la Catalina presentó los valores más altos de luminosidad, indicando que el grano presentaba una tonalidad más clara que los de las otras procedencias.

Igualmente, el análisis de varianza mostró efecto de la procedencia y el almacenamiento sobre la variable actividad enzimática, puesto que ningún valor-F es menor que el tabulado para 5\%, entonces todos los factores en estudio tienen efecto estadísticamente significativo sobre la actividad de la PPO con un nivel de confianza del 95,0\%, como se muestra en la tabla 3 . 
Tabla 3. Análisis de varianza para la actividad de la polifenil oxidasa de café crudo bajo tres fuentes de variación_procedencias y tiempos de almacenamiento.

\begin{tabular}{|c|c|c|c|c|c|}
\hline Fuente & $\begin{array}{l}\text { Suma de } \\
\text { Cuadrados }\end{array}$ & G libertad & $\begin{array}{c}\text { Cuadrados } \\
\text { medios del } \\
\text { error }\end{array}$ & $\begin{array}{l}\text { Razón F } \\
\text { calculada }\end{array}$ & $\begin{array}{l}\text { Razón F } \\
\text { tabulada }\end{array}$ \\
\hline \multicolumn{6}{|l|}{ Efectos Principales } \\
\hline A:ActPPO_Altur x procedencia & 16,0295 & 2 & 8,01476 & 1,66 & 3,89 \\
\hline $\begin{array}{c}\text { B: ActPPO x tiempo de } \\
\text { almacenamiento }\end{array}$ & 35,76 & 6 & 5,96 & 1,24 & 3 \\
\hline Residuos & 57,8171 & 12 & 4,8181 & & \\
\hline Total & 109,607 & 20 & & & \\
\hline
\end{tabular}

Fuente. Elaboración propia basada en los datos recolectados.

El método LSD indica que el café proveniente loración del grano como se propone en (30) de Naranjal es el de mayor actividad enzimá- y en (34) _ verde, amarillo, café y negro. Se tica inicial, y su tonalidad se hace más oscura reconfirma así que la ActPPO del café provedurante los dos primeros meses de almacena- niente de Naranjal durante el almacenamienmiento. Lo anterior confirma un deterioro del to es mayor que los cafés de las subestaciones café almacenado, según la secuencia de deco- Supía y la Catalina.

Tabla 4. Análisis de varianza para el contenido de lípidos de café crudo bajo tres fuentes de variación _procedencias y tiemposde almacenamiento.

\begin{tabular}{|c|c|c|c|c|c|}
\hline Fuente & $\begin{array}{c}\text { Suma de } \\
\text { Cuadrados }\end{array}$ & G libertad & $\begin{array}{c}\text { Cuadrados } \\
\text { medios del } \\
\text { error }\end{array}$ & Prueba-F & $\begin{array}{c}\text { Valor- } \\
\text { probabilidad }\end{array}$ \\
\hline Entre grupos & 1,86915 & 2 & 0,934575 & 9,67 & 0,0492 \\
\hline Intra grupos & 0,289962 & 3 & 0,0966542 & & \\
\hline Total (Corr.) & 2,15911 & 5 & & & \\
\hline
\end{tabular}

Fuente. Elaboración propia basada en los datos recolectados.

El aceite esencial o los lípidos, son los que conforman el aroma del café tostado, estos se encuentran en todo el endospermo del grano de café (32). El Coffea Arábica tiene entre el $12 \%$ y el $18 \%$ de lípidos en granos verdes como se describe en $(5,32)$. En este trabajo se encontró que los cafés recolectados y alma- cenados en la Catalina, tienen menor contenido de lípidos y presentan la mayor pérdida de estos durante el almacenamiento, pero están en el rango de los reportados en otros estudios. La razón Ftabulada, para el contenido de lípidos fue de 9,67, es el cociente entre el estimado entre-grupos y el estimado 
dentro-de-grupos. Puesto que el valor-p en la tabla ANOVA es menor que el tabulado para $5 \%$, existe una relación estadísticamente significativa entre Lipidos y Altura con un nivel de confianza del 95\%.

Como la correlación objetiva entre las variables fue tema de este trabajo en la tabla 5 se muestra que están correlacionadas inversamente. El rango de estos coeficientes de correlación va de -1 a +1 , y miden la fuerza de la relación lineal entre las variables. Los valores-p de la prueba de significancia estadística de las correlaciones estimadas menores de 0,05 indican correlaciones significativamente diferentes de cero como en este caso: 0,0320 , con un nivel de confianza del 95,0\%.

Tabla 5. Correlación entre los lípidos y la ActPPO para las tres procedencias y tiempos de almacenamiento.

\begin{tabular}{|c|c|}
\hline & Lípidos \\
\hline Lípidos & 1 \\
\hline ActPPO & $-0,8216$ \\
\hline
\end{tabular}

Fuente. Elaboración propia basada en los datos recolectados.

\section{Resultados del panel de evaluación sensorial}

El café procedente de Naranjal, presenta calificación aceptable a lo largo de los seis meses de almacenamiento. La pérdida de calidad durante el almacenamiento estuvo reflejada principalmente por los defectos detectados en tasa de: reposo, sucio y fermento. El amargo pronunciado es propio de cafés reposados (29, $30,34)$ y se presentó a partir del cuarto mes de almacenamiento, tiempo en el cual las actividades enzimáticas fueron menores, evidenciándose en los cafés marcadas alteraciones estructurales como lo propusieron en (34).
El café procedente de la Catalina presentó buena calidad para el tiempo cero (inicial), pero en el primer mes de almacenamiento presentó el defecto verde, y amargo pronunciado. El café de Supía, presentó un efecto sinérgico positivo para las muestras con pasilla, en los meses de almacenamiento $0,1 \mathrm{y}$ 4, excepto la mezcla 40-60, que sólo obtuvo buenas calificaciones hasta el primer mes de almacenamiento.

Para los meses 3 y 4 de almacenamiento el café excelso, consumo (100\%) y consumo-pasilla 90-10 presentaron buenas calidades, y defecto reposo para las mezclas consumo-pasilla 70-30 y 40-60.

Para todos los cafés, las características de aroma, intensidad del aroma y cuerpo presentaron buenas calificaciones, excepto para la mezcla consumo-pasilla 40-60, que presentó algunos altibajos para diferentes meses de almacenamiento.

Para el mes 5 solamente el café excelso presentó buenas cualidades, los otros tipos de café presentaron un marcado defecto reposo.

Para todos los cafés, las características de aroma, intensidad del aroma y cuerpo presentaron buenas calificaciones, excepto para la mezcla consumo-pasilla 40-60, que presentó algunos altibajos para diferentes meses de almacenamiento.

Se aprecia que el café procedente de naranjal presenta mejores cualidades organolépticas a través del tiempo y para estos la actividad enzimática es menor, como se muestra en la tabla 6. 
Tabla 6. Impresión Global de café crudo bajo tres fuentes de variación _procedencias y tiempos de almacenamiento.

\begin{tabular}{|c|c|c|c|c|c|c|c|}
\hline \multirow{2}{*}{ Procedencia } & \multicolumn{7}{|c|}{ Tiempo de almacenamiento/mes } \\
\cline { 2 - 9 } & 0 & 1 & 2 & 3 & 4 & 5 & 6 \\
\hline Café de Naranjal & 6 & 6 & 6 & 2 & 7 & 6 & 6 \\
\hline Café de Supía & 7 & 6 & 2 & 6 & 6 & 3 & 3 \\
\hline Café La Catalina & 6 & 6 & 6 & 6 & 6 & 6 & 6 \\
\hline
\end{tabular}

Fuente. Elaboración propia basada en los datos recolectados.

Para cada procedencia se aprecia una mayor tendencia al rojo para el café de Supía y al verde para el de la Catalina. El café de Naranjal se encuentra en un punto intermedio.

\section{Conclusiònes}

Estos resultados presentan una concordancia con estudios recientes desde una perspectiva fisiológica, donde se encuentran diferencias físicoquímicas y sensoriales basadas en la edafología $(6,7,9,10,14,38)$.

De todas formas, debe tenerse en cuenta que no existe equilibrio entre las características edafo climáticas entre los lugares de procedencia.

El contenido de lípidos es mayor en los cafés cultivados a mayor altura, igual que la actividad de la PPO y la diferencia en el color (AE). El análisis de varianza mostró el efecto de la procedencia sobre la variable luminosidad, durante el almacenamiento.

La actividad enzimática de la PFO de los cafés estudiados decrece con el tiempo, aunque no en forma severa, para los cafés de humedad menor al $11 \%$. Este comportamiento es más evidente al cabo de los seis meses de almacenamiento a $25^{\circ} \mathrm{C}$ y $70 \%$ de $\mathrm{HR}$, condiciones en donde la PFO presenta períodos de activación/inhibición los cuales indican que el grano está presentando modificaciones fisicoquímicas.

En conclusión, la actividad de la PPO es inversamente proporcional al contenido de lípidos, e inversamente proporcional a la calidad organoléptica (12).

La pérdida de calidad durante el almacenamiento estuvo reflejada principalmente por los defectos reposo, sucio y fermento detectados en tasa. $(33,36)$.

De lo encontrado en este trabajo se tiene que el almacenamiento de café verde almendra durante seis meses, a $24.5^{\circ} \mathrm{C}$ y $75 \% \mathrm{HR}$, afecta negativamente la calidad de la bebida, sobre todo las provenientes de mezclas consumo-pasilla, siendo más rápido este deterioro a niveles crecientes de pasilla.

Por medio de la colorimetría triestìmulo se diferencian exteriormente los cafés cultivados en diferentes altitudes y acorde con el tiempo de almacenamiento, específicamente en la coordenada a* del espacio CIELAB en donde este parámetro presenta valores menores para cafés cultivados a mayor altura. 
Se encontró un efecto significativo de la temperatura sobre el contenido de lípidos. Los resultados no mostraron diferencias significativas $(p<0,005)$ entre localidades con respecto al contenido lipídico total.

Agradecimientos: Los autores expresan su agradecimiento a todas las personas que colaboraron en la realización de la presente investigación.

Notas: Los métodos analíticos utilizados en este artículo fueron corroborados a través del macro proyecto "La investigación a partir de los recursos virtuales metodológicos y a través de las redes de los cursos y programas de la ECBTI-Tecnología del café" de la UNAD. Proyecto: Diagnostico y caracterización de las metodologías y técnicas diferenciadoras de la calidad en café en la cadena productiva del café.

Conflicto de Intereses: El trabajo no presenta conflicto de intereses de ninguna índole.

\section{Referencias}

1. CCI. Centro de Comercio Internacional.Calidad del café: guía del exportador, UNCTAD/OMC, Ginebra. 2002: 270-321.

2. Aguiar LC, Fazuoli TJ, Salva G, Favarin JL. Chemical diversity in coffee plants of Coffea canephora. Bragantia. 2005; 64: 577-582.

3. Fridell G. Coffee. John Wiley \& Sons. 2014.

4. Silva E, Mazzafera P, Brunini O, Sakai E, Arruda F, Mattoso L, Carvalho C, Pires, R. The influence of water management and environmental conditions on the chemical composition and beverage quality of coffee beans. Brazilian journal of plant physiolo- gy.2005; 17(2): 229-238.

5. Costa da Silva M, Castro H, Farnezi M, Pinto N, Andrade V, Silva E. Caracterização química e sensorial de cafés da chapada de minas, visando determinar a qualidade final do café de alguns municípios produtores. Ciência e Agrotecnologia. 2009; 33: 1782-1787. https://dx.doi.org/10.1590/S141370542009000700014 .

6. Neves BJ, Borem FM, Cirillo MA, Ribeiro MM, Alves $\mathrm{AA}$, Ramos $\mathrm{AH}$. Coffee quality and its interactions with environmental factors in Minas Gerais, Brazilian J. Agric. Sci. 2012; 4: 181-190.

7. Suárez SJC, Rodríguez BE, Duran BE. Efecto de las condiciones de cultivo, las características químicas del suelo y el manejo de grano en los atributos sensoriales de café (Coffea arabica L.) en taza. Acta Agronomica. 2015; 64: 342-348.

8. Alonso-Salces RM, Serra F, Reniero F, Héberger K. Botanical and geographical characterization of green coffee (Coffea arabica and Coffea canephora): chemometric evaluation of phenolic and methylxanthine contents. J. Agric Food Chem. 2009: 57(10):422435. doi: 10.1021/jf8037117. Epub 2009 Mar 19.

9. Scholz, M, Da Silva J, Garcia V, de Figuereido C, Kitzberger S. Sensory attributes and phisycalchemical characteristics of the coffee beverage from IAPAR cultivar. Coffee Science. 2013; 8: 6-16.

10. Cruz R, Morais S, Casal S. Mineral composition variability of coffees: A result of processing and production. In: Preddy V. (ed). Processing and Impact on Active Components in Food. Elsevier,The Netherlands.2015: 549-558.

11. Quideau S, Deffieux D, Douat-Casassus C, Pouységu L. Plant polyphenols: chemical properties, biological activities, and synthesis. Angew Chem Int Ed Engl. 2011; 50(3):586-621. doi: 10.1002/anie.201000044. Epub 2011 Jan 7.

12. Farah A, Monteiro M, Calado V, Franca AS, Trugo L.C. Correlation between cup quality and chemical 
attributes of Brazilian coffee. Food Chem. 2006; 98: 373-380.

13. Speer K, Kölling-Speer I. The lipid fraction of the coffee bean. Braz. J. Plant Physiol. 2006; 8 (1): 201216.

14. Pohl P, Stelmach E, Welma M, Szymezycha-Madeja A. Determination of the elemental composition of coffee using instrumental methods. Food Anal. Methods. 2013; 6: 598-613.

15. Bertrand B, Villarreal D, Laffargue A, Posada H, Lashermes P, Dussert $S$. Comparison of the effectiveness of fatty Acids, chlorogenic Acids, and elements for the chemometric discrimination of coffee (Coffea arabicaL.) varieties and growing origins. Journal of agricultural and food chemistry. 2008; 56(6):22732280 .

16. Hecimovic I, Belscak-Cvitanovic A, Horzic D, Komes D. Comparative study of polyphenols and caffeine in different coffee varieties affected by the degree of roasting. Food chemistry. 2011; 129: 991-1000.

17. Upadhyay R, Mohan-Rao LJ. An outlook on chlorogenic acids-occurrence, chemistry, technology, and biological activities. Critical Reviews in Food Science and Nutrition. 2013; 53(9): 968-984.

18. Cho AR, Park KW, Kim KM, Kim SY, Han J. Influence of roasting conditions on the antioxidant characteristics of Colombian Coffee (Coffee arabica) beans. Journal of Food Biochemnistry. (2013); 38(3). Doi:10.1111/jfbc. 12045.

19. Uman E. et al. The effect of bean origin and temperature on grinding roasted coffee. Sci. Rep. 2016; 6: 24483. doi: 10.1038/srep24483.

20. Wintgens J.N. Coffee: Growing, processing, sustainable production. A guidebook for growers, processors, traders and researchers. (2nded.), Wiley-VCH Verlag GmbH \& Co. KGaA, Weinheim, Germany (2012).

21. Gutiérrez H, De La Vara R. Análisis y diseño de experimentos. México: McGraw-Hill Interamericana; 2008.
22. CIE. (2016). Colorimetry, 3RD edition. Disponible en Commission Internationale de l'Eclairage: http:// www.cie.co.at, Acceso en: 22 de marzo de 2016.

23. Luo M, Cui G, Rigg B. The development of the CIE 2000 colour-difference formula: CIEDE2000. Color Research \& Aplication. 2001; 26(5): 340-350.

24. Mac Dougall D. Colour measurement of food: principles and practice: p. 3.1-3.12. En: D.B. MacDougall (Ed.). Colour in food. CRC Press, Boca Raton, FL; 2002.

25. Westland, S. Color. En: Paravina R, Powers JM. Esthetic color training in dentistry. Elsevier Mosby; 2004.

26. Gómez-Polo C, Portillo M, Lorenzo M, Vicente P, Galindo P, Martín A. Comparison of the CIELab and CIEDE2000 color difference formulas. The journal of prosthetic dentistry.2016; 115(1):65-70.

27. AOAC. Official methods of analysis. 18th ed, 2007 (on line) AOAC International, Gaithersburg, MD.

28. NTC. Normas técnicas colombianas. (2000). Bogotá, Colombia.

29. Puerta, G. Escala para la evaluación de la calidad de la bebida de café verde Coffea arabica, procesado por vía húmeda. Cenicafe. 1996; 47(4): 231- 234.

30. Borem F, Ribeiro F, Figueiredo L, Giomo G, Fortunato V, Izquierdo P. Evaluation of the sensory and color quality of coffee beans stored in hermetic packaging. Journal store products Research. 2013; 52:1-6.

31. Fridell G. Coffee. John Wiley \& Sons. 2014.

32. Speer K, Kölling-Speer I. The lipid fraction of the coffee bean. Braz. J. Plant Physiol. 2006; 18(1):201216.

33. Kreuml M, Majchrzak D, Ploederl B, Koenig J. Changes in sensory quality characteristics of coffee during storage. Food Science Nutrition. 2013; 1(4): 267- 272 .

34. Queiroz C, Lopes M, Fialho E, Valente-Mesquita V. Polyphenol oxidase: characteristics and me- 
chanisms of browning control. Food Reviews International. 2008; 24(4): 361-375. http://dx.doi. org/10.1080/87559120802089332.

35. Nebesny E, Budryn G. Evaluation of sensory attributes of coffee brews from robusta coffee roasted under different conditions. European Food Research and Technology. 2006; 224:159-165.

36. Rendón M, Garcia T, Bragagnolo N. Impact of chemical changes on the sensory characteristics of coffee beans during storage. Food Chemistry. 2014; 147: 279-286. https://doi.org/10.1016/j.foodchem.2013.09.123.

37. Selmar D, Bytof G, Knopp E. The storage of green coffee (Coffea arabica): Decrease of viability and changes of potential aroma precursors. Ann Bot. 2008; 101(1): 31-38.

38. Sunarharum W, Yuwono S, Pangestu W. \& Nadhiroh H. Physical and sensory quality of Java Arabica green coffee beans. IOP Conf. Series: Earth and Environmental Science. 2018;131. doi:10.1088/17551315/131/1/012018. 